ISSN: 0212-0267

DOI: http://dx.doi.org/ro.I420I/hedu20I837145164

\title{
INDIGENISMO, EDUCACIÓN COLONIAL Y ETNOEDUCACIÓN
}

\section{Indigenism, colonial education and etbno-education}

\author{
Martha Lucía Orozco Gómez \\ Universidad de Burgos \\ Correo-e: mlorozco@ubu.es
}

M. ${ }^{a}$ Dolores Fernández Malanda

Universidad de Burgos

Correo-e: fermal@ubu.es

Narda Dioselina Robayo Fique

Universidad Pedagógica Nacional (Bogotá)

Correo-e: nardarobayo@yahoo.es

Recepción: 8 de septiembre de 2017. Envío a informantes: 20 de septiembre de 2017 Aceptación definitiva: 2 de noviembre de 2017

REsumen: Con este trabajo se pretende abordar el indigenismo entendido como un movimiento político y cultural que defiende la identidad sociopolítica y el valor de la cultura de la América india, y que propone, a su vez, una alternativa en busca del bienestar colectivo. Esa alternativa surge del discurso postcolonial, crítico al desarrollo, y de las cosmovisiones de los pueblos originarios. Se denomina El Buen Vivir y se caracteriza por buscar otras vías al desarrollo más acordes con el respeto a la Pachamama, donde el ser humano forma un todo, siendo parte inherente del entorno natural y social que le rodea. De igual manera, se intenta mostrar cómo, a partir de los movimientos indigenistas, los pueblos originarios luchan por una educación propia que les permita mantener sus saberes ancestrales, amenazados en el presente por el neocolonialismo. Este trabajo surge a partir de la inquietud generada por las propias vivencias, resultado de la participación directa e in situ con diferentes comunidades indígenas que se encuentran en Abya Yala, y también a partir del conocimiento de diferentes investigaciones sobre el tema.

PAlabras Clave: movimientos indígenas; educación propia; cosmovisiones; buen vivir; pueblos originarios; neocolonización. 


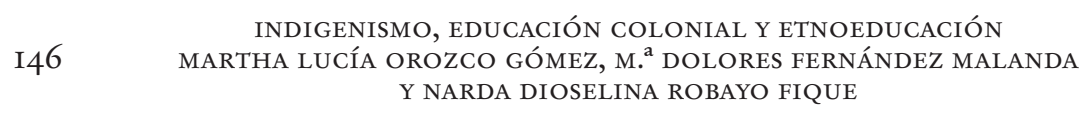

AвSTRACT: The purpose of this study is to address indigenism as a political and cultural movement which seeks to defend the socio-political identity and the cultural value of Indian Americans. This movement, which arose from post-colonial discourse that is crucial for development, and from the worldviews of indigenous communities, proposes an alternative in the search of collective welfare. This alternative is «El Buen Vivir» (The Good Living) which pursues other ways of development that are more in accordance with the respect for Pachamama (Mother Earth) and where human beings are considered an inherent part of the natural and socio environment that surrounds them. In the same way, this work aims to show how from indigenous movements, natives communities struggle for their own education that allows them to maintain their ancestral knowledge, threatened in the present by the new neo-colonialism. This work emerges from the concerns generated by the authors' own experiences and their knowledge from several research projects that involved direct participation with indigenous communities from Abya Yala.

KEY WORDS: indigenous movements; self-education; worldviews; good living; native communities; neo-colonialism.

$\mathrm{S}$

ON MUCHas las Cosas QUe nOS PREOCUPAN hoy EN día a la mayoría de personas, pero están controladas por un pequeño grupo movido principalmente por el deseo del poder y el tener. Entre esas preocupaciones se encuentra, en un nivel superior, el agotamiento de los recursos naturales y con ello la disminución acelerada de la diversidad del planeta, incluidos los animales, las plantas y la propia especie humana. En cuanto a esta última, aunque en número somos cada vez más, la diversidad disminuye por el mal entendimiento de lo que es el desarrollo, que está acabando con comunidades humanas que respetan la relación con el entorno y que son conscientes de la dependencia que tenemos de la madre tierra. En este aspecto deseamos centrar la atención en el presente artículo, independientemente de los objetivos de investigación que ha pretendido abordar el indigenismo entendido como un movimiento político y cultural que defiende la identidad sociopolítica y el valor de la cultura de la América india. Además de alternativas en busca del bienestar colectivo, es necesario reflexionar y visibilizar a unas comunidades que tienen mucho que decir y mucho que enseñarnos, con la esperanza de que aún estemos a tiempo.

Las investigadoras hemos tenido la oportunidad de convivir con diferentes comunidades indígenas, en diferentes partes de América, investigando bajo el modelo cualitativo y en ocasiones cuantitativo; investigaciones y vivencias que se han transformado en un profundo respeto a la sabiduría que encierran y que hemos querido plasmar en el trabajo que aquí presentamos con el propósito de contribuir a un mejor conocimiento de estas culturas y a la reflexión que mencionábamos hace un momento. En un primer apartado se intenta visibilizar y ofrecer una visión aproximada de la situación actual de los pueblos indígenas latinoamericanos, para seguidamente trabajar sobre el concepto del «Buen Vivir», que es una piedra angular para poder comprender el mundo indígena y la importancia 
INDIGENISMO, EDUCACIÓN COLONIAL Y ETNOEDUCACIÓN MARTHA LUCÍA OROZCO GÓMEZ, M. ${ }^{a}$ DOLORES FERNÁNDEZ MALANDA Y NARDA DIOSELINA ROBAYO FIQUE

de una relación armónica con todo lo que nos rodea. Teniendo esta visión, pasamos a un tercer apartado donde se analizan las consecuencias y el sufrimiento de unas personas sometidas a un proceso de aculturación y deculturación, citando un caso en concreto, pero que nos atrevemos a considerar extrapolable a la gran mayoría de comunidades. Finalizamos este breve recorrido analizando la educación, que constituye un elemento clave para la sobrevivencia y enriquecimiento mutuo de todos los saberes ancestrales.

\section{Una aproximación a la situación actual de los pueblos indígenas latinoamericanos}

Nuestro acercamiento a las comunidades indígenas Tikuna, Ashaninka, Quechua, Aymara, Wayúu, Lacandona o Tzotzil nos ha permitido conocer culturas que sobreviven, empoderando y fortaleciendo el sentimiento de comunidad, pese al impacto de la globalización, el individualismo, la cosificación y la despersonalización, pilares del capitalismo neoliberal.

Convivir por diferentes razones académicas y personales con estos pueblos nos ha ayudado a adquirir una enriquecedora visión del mundo a partir de la integración de cosmovisiones nuevas y reveladoras, caracterizadas todas ellas por una forma de ver y sentir el mundo más allá de nuestros enfoques etnocentristas, que nos ha permitido comprender la grandeza espiritual de culturas que, pese a dramáticos procesos de asimilación, han logrado sobrevivir'r.

La presencia de más de 45 millones de indígenas pertenecientes a más de 400 pueblos originarios distintos habla de la diversidad cultural como enseña del continente americano. Teniendo en cuenta esta realidad, acercarnos a estas culturas exige comprender su carácter multicultural, multiétnico y multilingüe.

Vivimos un momento histórico en el que los pueblos indígenas «interpelan» a la sociedad y a los Estados, pero el reconocimiento de la visión de las espiritualidades de estas comunidades es un tema pendiente y preocupante... Voces expertas señalan que todo ese «bagaje místico y mitológico» puede convertirse en «un fósil cultural»².

En el año 2006 sube al poder Evo Morales, el primer presidente indígena de la historia de Latinoamérica, emprendiéndose una profunda transformación de las instituciones del Estado, que presta especial atención a la descolonización de su historia, su cultura y su sociedad. Bolivia ha sido el primer país en ratificar la Declaración de las Naciones Unidas sobre los Derechos de los Pueblos Indígenas

\footnotetext{
IERnández Malanda, Dolores: «El "Sexto Sol” o el mito de la esperanza en el retorno de los tiempos nuevos: breve aproximación a la espiritualidad del Pacha Andino», en Cifuentes García, A. y Gómez Campelo, E. (coords.): Nuevas concepciones sobre el desarrollo en América Latina: elementos para el debate desde los Movimientos sociales y la Universidad, Burgos, Universidad de Burgos, 20I6, pp. II9-I32.

Carvalho Oliva, Homero: Seres sobrenaturales y mágicos de Bolivia, La Paz, Ministerio de Culturas y Turismo, 20II, p. 3.
} 
INDIGENISMO, EDUCACIÓN COLONIAL Y ETNOEDUCACIÓN
I48 MARTHA LUCÍA OROZCO GÓMEZ, M. ${ }^{a}$ DOLORES FERNÁNDEZ MALANDA
Y NARDA DIOSELINA ROBAYO FIQUE

(2007); esto marca un antes y un después para la constitución como naciones de estas comunidades.

En las Nuevas Constituciones de los Estados americanos -destacando las de Ecuador (2008) y de Bolivia (2009) - se contemplan y protegen por primera vez en su historia los derechos de los pueblos indígenas, con el compromiso de construir una ciudadanía que reconozca la igualdad y la diferencia ${ }^{3}$.

Las evocaciones políticas de los pueblos originarios en las diferentes constituciones deberían traducirse en leyes y políticas públicas sectoriales que dieran respuesta efectiva al reto que supone la multiculturalidad. Ahora bien, pese al protagonismo político de los pueblos indígenas, no ha habido resultados concretos en términos de reducción de la pobreza, por lo que aún se enfrentan a grandes obstáculos para obtener el pleno desarrollo de sus derechos y libertades a la hora de acceder a servicios como la educación, la justicia, los recursos naturales y la dotación de derechos fundamentales como el agua y la salud. Las acciones de lucha contra la pobreza no pueden olvidar incluir la interculturalidad para el reconocimiento de lo propio, que debe ser el eje para la promoción de acciones que lleven a la mejora de la calidad de vida. Resulta evidente que las profundas inequidades que han marcado su historia han conducido a la exclusión de la población indígena de los beneficios sociales y del desarrollo económico, condenándola en la mayoría de los casos a una situación de necesidad crónica.

A ello se suma la carga añadida de una globalización de sesgo neoliberal excluyente que provoca efectos similares a los del viejo colonialismo y neocolonialismo. Los que ocupan el poder económico siguen asociándose con la herencia cultural hispano-criolla de la colonia -más la de otras élites inmigrantes extranjeras-, identificándose con la actual corriente neoliberal globalizadora, por ser los que más se benefician de ella. En el otro extremo, los que permanecen desde hace siglos en la base de la pirámide social siguen relegados, cuando no oprimidos, por las viejas y nuevas fuerzas, asociados con las culturas tradicionales y locales, siendo con frecuencia los indígenas originarios. El principal obstáculo para un enfoque intercultural es la estructura general de poder, tanto en su dimensión económica como social.

La CEPAl (Comisión Económica para América Latina y Caribe), en su Informe del 2orı, afirma que el binomio indígena-pobreza es un complejo histórico, como lo fueron también las violaciones de Derechos Humanos fundamentales. Pese a que numerosas instituciones gubernamentales, programas, comisiones, se han encargado de "protegerlos» o «ayudarlos», siempre lo han hecho desde una relación subordinada4.

Los pueblos indígenas y originarios campesinos presentan los índices de mortalidad (destacando la materno-infantil) más altos en el orbe americano, además de problemas relacionados con la cobertura y el acceso a los servicios de salud.

Citarella Menardi, Luca; Zangari, Alessia y Campos Navarro, Roberto (eds.): YachaY TINKUY. Salud e interculturalidad en Bolivia y América Latina, La Paz, Editorial Gente Común, 20IO, p. 4 .

4 Ibidem, p. I4. 
INDIGENISMO, EDUCACIÓN COLONIAL Y ETNOEDUCACIÓN MARTHA LUCÍA OROZCO GÓMEZ, M. ${ }^{a}$ DOLORES FERNÁNDEZ MALANDA Y NARDA DIOSELINA ROBAYO FIQUE

De hecho, un alto porcentaje de ellos, variando según el país, se encuentran excluidos de su uso'.

En cuanto a la educación, sigue habiendo una población que varía de niños, niñas y adolescentes que no están matriculados o abandonan la escuela por el escaso nivel económico familiar, el elevado número de hijos e hijas en la familia, la baja escolaridad de los padres, y por causas propias del sistema educativo, como la limitación de profesores y de servicios de apoyo en los centros, el elevado número de alumnos y alumnas por aula, la falta de formación de los maestros/as o la oferta educativa incompleta 6 .

Pese a la crudeza de los datos, las áreas de la Salud y de la Educación siguen siendo líneas prioritarias en la Hoja de Ruta de las Agendas Públicas para muchos Estados americanos.

La cuestión es qué entienden los pueblos originarios por salud y por educación. Para ellos, y esto implica la plena conciencia de la riqueza de la alteridad, de la cultura de ese «otro», es un asunto de dimensión colectiva, relacionado con el entorno ecológico, sociocultural, económico, político, espiritual y emocional.

Por este motivo no debemos olvidar, y más en medio de un contexto multicultural de amplio espectro, que las personas que asisten a los centros de salud, a las escuelas, a las instituciones públicas llevan sus propias historias, sus cosmovisiones, formas de interpretar el mundo, la vida comunitaria, los procesos de salud y de enfermedad, y estos no siempre coinciden con la lógica oficial. De ahí la imperiosa necesidad de diseñar e implementar políticas públicas aptas para esta realidad, con un auténtico enfoque intercultural que articule y complemente el saber tradicional con el oficial7.

\section{Hacia una comprensión del mundo indígena. El regreso al «Buen Vivir»}

Durante siglos, el mundo indígena ha sido «asimilado» a la estructura social dominante de las culturas europeas. Es un mundo amplio, extenso, que comprende

Según datos extraídos de diferentes fuentes, en países como México la mortalidad infantil indígena es un $60 \%$ superior a la del resto de la población infantil; en Panamá, 44,6 de cada mil niños/as indígenas nacidos mueren, en Guatemala 55 y en Bolivia 77 (CEPAL, 20I4). En la población indígena de Colombia se presenta una de las más altas tasas de mortalidad infantil del mundo: un promedio de 250 de cada mil niños/as en las comunidades de Paeces en el Cauca, Awá en Nariño y Emberá en Antioquía fallecen antes de los 5 años. Las causas en muchos de los casos se vinculan al envenenamiento de las aguas por el extractivismo. CARDona, J. et al.: «Las condiciones de vida de los niños del resguardo indígena Cañamomo-Comaprieta (Colombia)», Revista Médica UIS, Santander-Colombia, vol. 27, n. ${ }^{\circ} 2$ (2014), pp. 29-39.

6 García Rodríguez, Mara: Hacia el empoderamiento emocional de los adolescentes trabajadores del Centro Minero "Yachay Mosoj», Programa de Educación Afectivo Sexual (2010-2013), tesis doctoral inédita, Universidad de Burgos, 2015. La CEPAL nos indica que casi el $20 \%$ de los niños y niñas indígenas de 6 a II años aún no están escolarizados en países como Guatemala, El Salvador o Nicaragua, por citar alguna de sus estadísticas respecto a la educación de los pueblos indígenas y afrodescendientes en Latinoamérica (2014).

7 Fernández Malanda, Dolores: op. cit., pp. II9-132. 
a cientos de pueblos, cada uno con sus propias características, lenguas, costumbres. Todos ellos comparten, sin embargo, un principio de vida que organiza su modo de existir y de estar en comunión con el cosmos. Este principio fundamental es lo que se ha dado en llamar «la dualidad complementaria», basada en sistemas de reciprocidad y de solidaridad existencial, algo que lo contrapone a nuestro mundo individualista y que constituye el eje vertebrador de una filosofía de vida basada en los saberes ancestrales, conocida como Sumak Kawsay, Sumak Qamaña o «Buen Vivir» ${ }^{8}$. Se trata de un principio rector que ha sobrevivido a siglos de avasallamiento, subsistiendo en el corazón, alimentando ritos, manteniendo la vivencia de lo espiritual, la construcción de la identidad desde los caminos de la complementariedad?.

Sin comprender esta dualidad no podemos entender al indígena; todo gira en torno a la idea de que uno se debe a otro, porque ese otro se hace parte de uno mismo, y eso explica la importancia dentro de estas culturas milenarias del cuidado, el respeto y la aceptación del otro, algo que les ha hecho y hace vulnerables a las ideologías depredadoras de los antiguos y los nuevos colonizadores.

La estructura social se basa en unos sistemas de permuta que constituyen uno de los fundamentos primordiales de estas cosmovisiones ${ }^{10}$. Son paradigmas incomprensibles para nosotros, al estar basados en el absoluto desprendimiento de los bienes materiales e inmateriales.

El espíritu comunitario es el eje que vertebra la organización social, entendiéndose la comunidad como un nosotros complementario. La vida individual es insuficiente y complicada, pues «el ser de la comunidad se proyecta en el tiempo donde está dando y recibiendo de modo gradual y alternativamente... la reciprocidad no pertenece ni al donante ni al otro, sino de forma igual a todos los comunarios» $»^{\mathrm{II}}$.

Pero la relación del nosotros no solo se circunscribe a la comunión de las personas entre sí; va más allá, hasta la comunión con la Pachamama, la naturaleza, y hasta el reino de las divinidades que la protegen. Sobre el vínculo espiritual con la naturaleza, el hombre establece un diálogo con ella, porque sin ese diálogo quedaría perdido, al depender de ella todo su existir. El encuentro con la naturaleza fortalece el nosotros.

La dimensión divina también forma parte de la comunidad y de la naturaleza, compartiendo los mismos menesteres, cotidianeidad, el «nosotros trascendental», sin diferenciar espacios de convivencia: representa el encuentro sagrado y el tiempo presente de amor y entrega.

\footnotetext{
8 Bascopé C., Víctor: Espiritualidad originaria en el Pacha Andino. Aproximaciones teológicas, Cochabamba, Edit. Verbo Divino, 2013 (3. ${ }^{a}$ ed.).

9 Platt, Tristán: «Espejos y maíz: temas de la estructura simbólica andina», Cuadernos de Investigación y Promoción del Campesinado, n. ${ }^{\circ}$ I4 (1976), p. 27.

ro Amadio, M. y D’Emilio, A. L.: Cultura. Material de apoyo a la Formación Docente en Educación intercultural y Bilingüe, La Paz, UNICEF, I993, p. II2.

" Nina Baltasar, Esteban: La reciprocidad. Ethos de la cultura aymara, Cochabamba, Publicaciones de la Universidad Católica Boliviana, I991, p. 139.
} 
INDIGENISMO, EDUCACIÓN COLONIAL Y ETNOEDUCACIÓN MARTHA LUCÍA OROZCO GÓMEZ, M. ${ }^{a}$ DOLORES FERNÁNDEZ MALANDA Y NARDA DIOSELINA ROBAYO FIQUE

Las comunidades indígenas, en su concepción religiosa y ética, basan sus creencias acerca del origen en los mitos y las leyendas, de las cuales nace el profundo respeto por la naturaleza y por toda manifestación de vida, puesto que la consideran sagrada por ser una creación de los dioses para la Humanidad.

Derivado de la profunda reflexión de las comunidades indígenas latinoamericanas en torno a cómo enlazar -en un mundo marcado por el capitalismo neoliberal- cosmovisiones basadas en la triple unión del hombre con sus congéneres, con la naturaleza y con lo espiritual, nace el «Buen Vivir», entendido como

filosofía de vida que abre sus puertas para construir un proyecto liberador y tolerante, sin prejuicios ni dogmas. Un proyecto que, al haber sumado muchas historias de luchas, de resistencias y de propuestas de cambio, al nutrirse de experiencias nacionales e internacionales, se posiciona como punto de partida para construir democráticamente sociedades democráticas ${ }^{12}$.

$\mathrm{El}$ «Buen vivir» se sostiene sobre pilares de resistencia y de cambio frente al actual modelo hegemónico, oponiendo a este visiones armoniosas biocéntricas que, pese a las fuerzas que las contrarrestan e intentan violentar, permiten que algunos pueblos preserven su esencia al edificar su día a día en tres principios fundamentales, que articulan el espíritu de todas las naciones indígenas: territorio-comunidadidentidad. El territorio, que surge como concepto primordial, es históricamente una de las reivindicaciones de mayor importancia, pues remite tanto a la lucha por la propiedad como a la defensa del entorno vital. El espacio vital, por lo tanto, es entendido en una doble dimensión: material (la tierra) y simbólica (apropiaciones sociales), y es construido por la comunidad, unidad básica de socialización de todo ser humano. La comunidad, a su vez, está determinada por las particularidades de cada uno de sus integrantes, y, finalmente, produce una identidad colectiva ${ }^{13}$.

El Sumak Kawsay es un modelo alternativo al desarrollo que nos interpela sobre la necesidad de generar prácticas y pautas sociales basadas en la reciprocidad, la armonía y la solidaridad como códigos de conducta éticos con las personas y con la naturaleza, entendiendo el territorio y su contenido como algo que no nos pertenece; nuestra misión es nutrirlo y preservarlo para las futuras generaciones.

Cuando se produce el quebrantamiento de los espacios y el incumplimiento de las funciones establecidas para cada ser, se genera de modo inminente un proceso «desarmonizante» en forma de todo tipo de catástrofes. Lo importante en esos momentos es conocer los hechos que han ocasionado la ruptura de las relaciones armoniosas del Pacha o principio de armonía universal, con el consecuente desequilibrio universal, puesto que en la sabiduría ancestral existe el conocimiento de los medios para la restauración de la armonía del Pacha, y estos medios restauradores son los ritos. Al respecto, Virreira afirma que

${ }_{12}$ Acosta, Alberto: El Buen Vivir: Sumak Kawsay, una oportunidad para imaginar otros mundos, Barcelona, Icaria, 20I3, p. I8.

13 GARzón PÉRez, Luis Alfonso: Controversia entre Sumak Karwsay y crematística: una lectura desde el pueblo Tikuna, tesis doctoral inédita, Universidad de Burgos, 2016. 
conservar invariable la estructura jurídica y moral es sinónimo de conservar la vigencia del orden en el universo. Cuando el orden es vulnerable sobreviene una catástrofe que pone fin a una era temporal. El nuevo orden debe ser descubierto y pacientemente construido para posibilitar la vida futura ${ }^{\mathrm{I} 4}$.

Esto explica que, en todas sus cosmovisiones, la desaparición de grandes imperios como el Tolteca, el Olmeca, el Mexica, el Inca, el Tiahuanaco o el Maya, por citar algunos, así como los cruentos tiempos de la Colonia o el devastador efecto de las multinacionales, no dejen de ser el fruto y la consecuencia de momentos en los que el hombre quebrantó o quebranta, por diferentes motivos, su vínculo con la Pachamama y con los seres sobrenaturales (espíritus y dioses tutelares) que protegen toda la creación.

En América, a diferencia de lo sucedido en otros continentes, se produce un corte «a cuchillo de una trascendencia cataclísmica a partir de la llegada de los europeos al hemisferio, lo que marca una especie de grieta histórica de extraordinaria complejidad, crucial para desentrañar los problemas de nuestra identidad y autoidentificación» ${ }^{15}$. El «Quinto Sol» de las tradiciones andinas, mayas, mexicas y amazónicas, que debía haber brillado con todo su esplendor en la historia de la humanidad, se oscurece como consecuencia de la invasión genocida, resurgiendo a partir de los años 30 del siglo xx con el nuevo pensamiento que en la clandestinidad van articulando los pueblos indígenas y originarios, concretado en mayo de 1945 con el Primer Congreso Indigenal Boliviano, celebrado en La Paz, donde se dieron cita pueblos andinos y amazónicos.

En la medida en que los pueblos indígenas y originarios fueron pensándose a sí mismos, se afirmó la idea de que el mundo prehispánico era una sociedad perfecta que fue devastada por la codicia europea. Restituir el pasado originario se constituyó en la base de una ideología que se hizo transversal a las reivindicaciones sociales.

Los años sesenta traen un movimiento de pensadores, escritores, sociólogos, antropólogos, teólogos, conocidos como los «indigenistas» o «indianistas», que descubren el valor de "ser indio", de ser «indígena», "originario», y que defienden con firmeza que los pueblos debían vindicarse desde su identidad como tales.

El «Sexto Sol», el tiempo anunciado y esperado, el tiempo de esperanza de Atawalpa, de Rumiñawi, de Tupaq Amaru, de Tupaq Katari, surge con el Primer Levantamiento Indígena del Ecuador en I990; con la Primera Marcha Indígena del Oriente Boliviano en I990; con la Insurgencia de Chiapas en I994; con todas las Marchas por la Vida, por la Tierra y el Territorio, por los Recursos Naturales, por el Agua, que movilizaron a las naciones bolivianas en la Marcha de las comunidades indígenas del sur de Colombia en el 2008, o con las últimas movilizaciones por el territorio indígena y Parque Nacional Isiboro-Secure (TIPNIS) en Bolivia.

14 Virreira Guzmán, Jaime: Sexto Sol y Octavo Día, La Paz, Centro de Promoción Social del Arzobispado, 1982, p. 63.

is Mesa Gisbert, C: La sirena y el charango. Ensayo sobre el mestizaje, La Paz, Fundación Comunidad, 2014 (3. ${ }^{a}$ ed.), p. I6. 
INDIGENISMO, EDUCACIÓN COLONIAL Y ETNOEDUCACIÓN MARTHA LUCÍA OROZCO GÓMEZ, M. ${ }^{a}$ DOLORES FERNÁNDEZ MALANDA

Y NARDA DIOSELINA ROBAYO FIQUE

\section{Aculturación y deculturación. Una breve revisión del proceso de asimilación de las comunidades indígenas de Colombia}

Para analizar este aspecto es fundamental conocer las relaciones establecidas entre el Estado, la Iglesia y la problemática de la población indígena, partiendo del contexto de las ideologías políticas imperantes en la época, que contribuyeron a consolidar la hegemonía de la Iglesia católica sobre la educación.

La metrópoli española se comprometió, desde el comienzo de la Conquista, a evangelizar a la población nativa asentada en las tierras conquistadas, a velar por la salud espiritual de sus gentes y a afianzar la prosperidad material de la Iglesia, en quien se delegó la administración y dirección de los hospitales y las instituciones educacionales, lo que la convirtió en uno de los más importantes agentes del poder civil ${ }^{16}$.

Un caso significativo y que se replica es el de Colombia. La sociedad nacional decimonónica estaba inmersa en los vaivenes de políticas liberales y conservadoras, responsables de conflictos y guerras. El excesivo poder de la Iglesia incomodaba a muchos sectores de la población, que veían en ella un obstáculo para el desarrollo económico del país. En medio de estas discrepancias, el Gobierno Central dictó en 1850 un conjunto de normas anticlericales, entre ellas la de derogar la autoridad de la Iglesia sobre la educación, con la consiguiente expulsión de las órdenes que desempeñaban esta misión, de modo especial la orden de los jesuitas.

Con la Constitución de I886, de corte conservador, se restituyó a la Iglesia la facultad de dirigir la vida de la sociedad y el control de la educación. Esto ocurre inmediatamente después de haberse firmado el Concordato entre la Santa Sede y el Estado en I887, mediante el cual el Estado le concede a la Iglesia católica plena autonomía y le encarga la educación de la nación, que deberá regirse por los dogmas y moral católicos. Colombia firmó en i888 la primera convención con las Misiones católicas para la colonización y cristianización de la periferia del país, lo cual motivó la difusión de órdenes religiosas encaminadas a civilizar a los indígenas bajo la visión sociocultural de la sociedad nacional ${ }^{17}$. Los principales encargados de estas Misiones fueron las órdenes de los capuchinos y los franciscanos, que en el caso del Trapecio amazónico crearon nuevos establecimientos educativos para las comunidades tikunas ${ }^{18}$.

Dos años más tarde, la ley 89 sobre indígenas deja en manos de las Misiones la protección de los indígenas no civilizados, y el Estado asume el patrocinio de los indígenas occidentalizados. Pero en I892, ante la incapacidad de gobernar todo el país, el Estado le confiere a la Iglesia la administración general de las regiones periféricas, destinando todos los recursos necesarios para el mantenimiento de las escuelas, incluyendo infraestructuras, profesores y materiales escolares.

16 Helg, Aline: La educación en Colombia: 19I8-1957, Bogotá, Editorial Plaza y Janés-upN, 200I.

${ }_{17}$ Ibidem.

18 El registro de estos establecimientos lo encontramos en los documentos oficiales del Centro de Promoción Indígena de la Amazonía Colombiana (CEProiac). 
Con tal responsabilidad, las Misiones religiosas adquieren, entre otras facultades, la de imponer a los nativos la escolarización, la enseñanza del castellano y la conversión a la religión católica; herramientas indispensables para lograr el propósito de cambiar el paradigma de la cultura indígena por el modelo occidental.

Podemos indicar que en las últimas décadas del siglo xix la Iglesia de nuevo estaba presente en todo el territorio con una notable fuerza política, lo que le permitía intervenir en todos los aspectos de la vida de la sociedad, que obedecía y respetaba mayoritariamente sus decisiones, ejerciendo el control de la educación, ya que la casi totalidad de los establecimientos educativos eran manejados por religiosos ${ }^{19}$.

Como se puede observar, la misión principal de la Iglesia católica no fue de carácter espiritual, sino que comprendió la sujeción de todos los elementos de las culturas indígenas al paradigma occidental. Lo hizo desde un adoctrinamiento que partió de la imposición de la lengua castellana (con la consiguiente prohibición de la propia) y desde una educación auspiciada por el Estado, que significó la aculturación y deculturación de los pueblos nativos sometidos y la exclusión de sus expresiones culturales ${ }^{20}$. Pero la firmeza de las creencias de los indígenas y las luchas de los movimientos de resistencia no permitieron arrasar totalmente sus saberes ancestrales, lo que dio paso, en la mayoría de los casos, a la formación de culturas sincréticas o híbridas ${ }^{21}$.

En el caso del Estado colombiano, una vez terminado su litigio con Perú en I933, se procedió a la nacionalización de los pueblos originarios ubicados en el Trapecio amazónico, considerados como los últimos baluartes indígenas. Esta nacionalización se hizo con el propósito de incluirlos en la sociedad mayoritaria, y a la vez cimentar la soberanía en estos territorios, más que con el objeto de respetar y procurar la conservación de su propia cultura.

En 1973 el Estado y la Santa Sede establecen nuevas relaciones, y la Iglesia es relevada de sus funciones educativas. Es entonces cuando el quehacer educativo es asumido por el poder civil en Colombia, aunque la Iglesia continúa participando en la difusión de la educación oficial entre las comunidades indígenas mediante el sistema denominado «Educación contratada». Este sistema opera principalmente en los antiguos territorios nacionales (intendencias y comisarías elevados a Departamentos en el nuevo orden territorial de i991), facilitando la educación en zonas alejadas y de difícil acceso, entre las que se encuentra el Departamento del Amazonas ${ }^{22}$.

El sistema escolar, la metodología y los contenidos implantados por los misioneros y por el Estado, al violentar el esquema cultural de las comunidades indígenas, motivó su resistencia. Estas comunidades reclamaron, a través de sus organizaciones sociales, una educación acorde a su visión, necesidades, usos y

19 Ferroni, E. y Jiménez, G.: Mundo moderno, Bogotá, Libros \& libres, 1994.

20 González, C. y Arroyave, L.: Historia de América, Medellín, Bedout, 1975.

${ }_{21}$ SÁnchez, E. y Molina, H.: Documentos para la historia del movimiento indígena colombiano contemporáneo, Bogotá, Ministerio de Cultura, 20 oro.

22 Sinchi: Misión. Colombia amazónica, Bogotá, Sinchi, 2009. 
INDIGENISMO, EDUCACIÓN COLONIAL Y ETNOEDUCACIÓN MARTHA LUCÍA OROZCO GÓMEZ, M. ${ }^{a}$ DOLORES FERNÁNDEZ MALANDA Y NARDA DIOSELINA ROBAYO FIQUE

costumbres, expectativas y particularidades culturales; después de varios años de luchas y acciones a nivel nacional e internacional, las solicitudes encontraron eco en la Nueva Constitución Política de i991.

Una vez que el Estado reconoce, con la Nueva Constitución Política, la diversidad étnica y cultural de la Nación colombiana, y establece que el idioma oficial de Colombia es el castellano, y que las lenguas y dialectos de los grupos étnicos son también oficiales en sus territorios, la enseñanza que se imparte en las comunidades con lenguas propias empieza a ser bilingüe. Con la Ley General de Educación de 1994 se reglamenta que «la educación para los pueblos indígenas, tendrá en cuenta criterios de integralidad, interculturalidad y diversidad lingüística».

Sin embargo, es importante indicar que las propias vivencias y las experiencias que se han llevado a cabo no son todo lo satisfactorias que deberían. Por un lado, porque en la capacitación de los docentes, de manera general, no se introduce la formación necesaria en materia de diversidad cultural, y mucho menos de los diferentes dialectos y lenguas existentes, por lo que en el momento de ejercer su profesión desconocen los diversos grupos étnicos existentes en cada país. Se perfila así un maestro con características y competencias para una actuación uniforme en cualquier contexto, desconociendo lo pluriétnico y multicultural, que requiere docentes con características específicas para responder a las necesidades de los pueblos indígenas. Por otro lado, se ha constatado que en la práctica la lengua vehicular es el castellano, a pesar de que muchos indígenas, en el mejor de los casos, la hablan mal, y otros ni siquiera la entienden; además, en muchas regiones se utiliza como segunda lengua el inglés. Los maestros no conocen ni tienen la más mínima formación en las variadas lenguas existentes en los diferentes territorios, lo que dificulta desde todo punto de vista la equidad en el proceso de enseñanza-aprendizaje $\mathrm{e}^{23}$.

\section{El encuentro entre la educación oficial y la educación propia}

Los Estados americanos han sido concebidos dentro de una visión eurocéntrica. Ello explica, o justifica de alguna manera, que en la mayoría de las ocasiones hayan impuesto arbitrariamente a las comunidades indígenas esa misma visión, sin tener en cuenta su consentimiento y aprovechándose de su vulnerabilidad. Se trata, en palabras de Restrepo, de «un intento de integración impuesto por una cultura dominante», que convierte en «subalternas» a esas comunidades ${ }^{24}$.

Hablamos del encuentro de dos culturas con cosmovisiones totalmente opuestas y en desigualdad de condiciones: una representada por el Estado, la sociedad

${ }_{23}$ Se pueden consultar varias experiencias educativas que dan cuenta de las dificultades que se han presentado a la hora de poderlas llevar a cabo en América Latina en el estudio coordinado por Daniel Mato, Diversidad Cultural e Interculturalidad en Educación superior, financiado por la UNESCO y el IESALC (2008).

24 Restrepo Orrego, Carlos E.: Apropiación indebida de recursos genéticos, biodiversidad y conocimientos tradicionales: "biopiratería», Bogotá, Universidad Externado de Colombia, 2006, p. 153 . 
mayoritaria y la Iglesia católica que, a través de la religión, la lengua y la educación domina a la otra, representada por los pueblos indígenas, que expresan su acervo cultural a través de la oralidad, los saberes ancestrales y una mitología y ritualística basados en el amor al territorio y a los antepasados, todo ello desde una perspectiva muy lejana al individualismo propio del Occidente hegemónico.

Debido a la imposición del paradigma occidental, a la relación con personas extrañas a su cultura y por efecto de la globalización, las comunidades indígenas han entrado en contacto con otros modelos de cultura y han sido impactadas por los numerosos cambios ocurridos. Su relación con el mundo calificado como civilizado las ha puesto en emergencia y alerta para defender sus culturas, bienes materiales y espirituales y el derecho a existir como pueblos diferentes, encontrando en la educación una herramienta fundamental para asimilar las nuevas situaciones y continuar manteniendo su identidad y cultura propias como comunidades ancestrales renovadas.

Las organizaciones sociales indígenas cuestionan el modelo de educación oficial impuesto por los Estados nacionales y reclaman autonomía para practicar una educación propia, acorde con su cosmovisión, usos y costumbres, necesidades y expectativas. Para tal fin, dichas organizaciones se han reunido en diversas ocasiones con el propósito de debatir y llegar a acuerdos sobre temas relacionados con asuntos pedagógicos, metodologías y estrategias conducentes al fortalecimiento y calidad de la educación indígena. Los acuerdos se han ido concretando y canalizando a través de diversos organismos ${ }^{25}$. Estas políticas de educación propia se han generalizando a nivel nacional en todos los Estados.

La educación propia para los pueblos indígenas constituye un proceso integral de rescate y fortalecimiento de la lengua materna; de recuperación y recreación de valores culturales, tradiciones, mitos, leyendas y manifestaciones culturales como la danza o el canto, y de revitalización de los saberes ancestrales. También requiere

la identidad cultural y formas propias de organización jurídica y sociopolítica de los pueblos indígenas [...] para lograr un buen vivir comunitario centrado en la unidad, diálogo, reciprocidad, capacidad para proyectarse y articularse a otras sociedades respetando los derechos de todos, hacia la construcción de sociedades plurales y equitativas [...]. Es un proceso de enseñanza aprendizaje que se adquiere desde antes del nacimiento hasta después de la muerte; que se construye y valida por cada pueblo y se concreta en su respectivo Plan de Vida y es promovido por los sabedores, el núcleo familiar y mayores en general ${ }^{26}$.

Este modelo de educación se ha venido perfeccionando a través del tiempo, y ha sido producto de un arduo trabajo desarrollado colectivamente por las organizaciones indígenas, basándose en sus experiencias, creencias y cosmovisiones,

${ }_{25}$ Congreso Nacional Indígena (México), Municipios Autónomos Rebeldes Zapatistas (México), Consejo Regional de Indígenas del Cauca CRIC (Colombia) o el Comité de Unidad Campesina (Guatemala).

${ }^{26}$ CONTCEPI: Perfil del sistema educativo indígena propio, Bogotá, SEIP, 20I3, p. 36. 
INDIGENISMO, EDUCACIÓN COLONIAL Y ETNOEDUCACIÓN MARTHA LUCÍA OROZCO GÓMEZ, M. ${ }^{a}$ DOLORES FERNÁNDEZ MALANDA Y NARDA DIOSELINA ROBAYO FIQUE

y que les ha servido como eje fundamental para revitalizar sus culturas, mantener su identidad y subsistir como pueblos diferentes, al mismo tiempo que ha supuesto un soporte fundamental para articular sus luchas y reclamaciones por la defensa del territorio y de su autonomía.

Los movimientos indígenas que abogaban por el derecho a la educación propia se fortalecieron en los años 8o, cuando las organizaciones que luchaban por la defensa del territorio y la autonomía también empezaron a reclamar públicamente el derecho a la cultura propia. Debido a los reclamos de las comunidades indígenas de este derecho y a su reconocimiento, tanto por el Estado colombiano como por las instituciones internacionales, se diseña el modelo de la Etnoeducación.

Para cumplir con este requisito se han fundado centros de educación donde se forman maestros especializados en Etnoeducación. Se pueden mencionar ejemplos como la Universidad Autónoma Indígena Intercultural (UAIIN) que funciona en la ciudad de Popayán, donde se prepara a los alumnos en Educación Bilingüe e Intercultural para que posteriormente puedan administrar y atender las escuelas y colegios de las comunidades indígenas.

Después de varias acciones, en 1982 tiene lugar el Primer Encuentro Nacional de Etnoeducación, evento que fue convocado y coordinado por la Organización Nacional Indígena Colombiana (ONIC) y el Ministerio de Educación Nacional (MEN) con el propósito de avanzar conjuntamente en el diseño de los programas educativos. Pero a pesar de esta importante acción reivindicativa, el MEN continuó determinando que la educación en grupos étnicos estará orientada por los principios y fines establecidos en la Ley General de Educación de 1994.

Por tal razón los pueblos indígenas continuaron sus movilizaciones por el derecho a una educación diferenciada y autónoma. Después de los encuentros y concertaciones entre el MEN y la ONIC durante los tres primeros años del siglo XxI, surge la Mesa Nacional de Concertación de la Política Educativa de los Pueblos Indígenas de Colombia, entendida como el espacio donde se construyen las políticas públicas de educación para los pueblos indígenas ${ }^{27}$.

Con base en estos antecedentes, nace el Sistema Educativo Indígena Propio (SEIP) como mecanismo de concreción de la Educación Propia, orientado también por la legislación nacional e internacional, entre la que podemos citar la declaración de las Naciones Unidas sobre los Derechos de los pueblos indígenas (2007) y el convenio I69 de la OIT. El SEIP se vertebra sobre la familia debido a que es en su seno donde los niños y las niñas reciben conocimientos primordiales para la supervivencia de los saberes ancestrales y para la convivencia pacífica de la comunidad. Estos conocimientos se adquieren en estrecha relación con la naturaleza, a través de vivencias que son transmitidas oralmente por los adultos y ancianos con la intercesión de las «autoridades mayores» y los espíritus de los ancestros.

Como instrumento guía para desarrollar el Proyecto de Educación Propia, el SEIP propuso el diseño del Proyecto Educativo Comunitario (PEC) como un componente de concreción de la Educación Propia. Una vez que las condiciones de

${ }_{27}$ Ibidem. 
desarrollo educativo se consolidaron en las comunidades a nivel local y regional, el movimiento educativo propuso el PEC en cada institución educativa. Se trata de una propuesta alternativa a los Proyectos Educativos Institucionales (PEI) establecidos por el MEN, como desarrollo de la Ley general de Educación, para todo el país. Esto se hace con el propósito de restaurar la educación mediante el fortalecimiento de la capacidad de cada centro educativo, posibilitando así una relativa autonomía de cada institución educativa, dando mayor participación a la comunidad y atendiendo las particularidades de cada grupo social ${ }^{28}$.

Los PEC son una propuesta diseñada por las comunidades indígenas, en la cual se plasma todo el contenido de la política educativa de cada pueblo indígena, y a su vez constituyen una herramienta importante en la construcción de los «Planes de vida», en los que se inspiran la estructura y el funcionamiento de cada comunidad, conforme a su cosmovisión y atendiendo a su problemática y expectativas. Las comunidades ejercen así su autonomía con la participación de sus miembros y toman sus propias decisiones, compartiendo los saberes con otras culturas.

El contacto de los pueblos indígenas con la cultura de Occidente les ha permitido conocer el proceso vertiginoso que ha seguido la humanidad en su progreso, en el cual están inmersos como parte importante de la sociedad mayoritaria. Saben que deben prepararse para afrontar los retos que les presentan la modernización y la globalización, que han colonizado todos los rincones de la tierra imponiendo una visión de mundo diferente a la visión indígena. Por tanto, el SEIP «propone una educación que prepare a los individuos para afrontar los retos del mundo actual» $»^{29}$.

Esta formación integral es importante no solamente en los infantes, sino también para los jóvenes. Todo este conjunto de conocimientos ancestrales es transmitido de forma oral, proceso que se ha de articular con la escuela, dando continuidad a los aprendizajes familiares y comunitarios, relación que es fundamental para el logro de la calidad del proceso educativo. Se considera la familia como la célula fundamental donde se origina la organización de la sociedad ${ }^{30}$.

El papel educativo de la familia se especifica en el apartado denominado «Semillas de Vida», el cual es definido como el «sistema de interacción dinámica y reafirmación espiritual, cultural y lingüística desde la cosmovisión propia, la vivencia infantil y el contacto estrecho entre los padres, los mayores y los menores, que constituyen la base de los futuros comportamientos y responsabilidades socioculturales ${ }^{31}$. Este documento recoge, entre otras cuestiones, la importancia de preservar los saberes, usos y costumbres y las dinámicas de socialización e

28 Bolaños, Graciela: «Ustedes y nosotros, diferentes mas no inferiores, la construcción de un proyecto educativo indígena en Colombia», Revista Educación y Pedagogía, Medellín, vol. XIX, núm. 48 (2007), p. 59.

29 CONTCEPI: op. cit., p. I3.

30 Robayo Fique, Narda D.: Prácticas de conservación utilizadas en agricultura itinerante en la comunidad indígena Tikuna de San Francisco de Asis, municipio de Puerto Nariño, Departamento de Amazonas, Colombia, tesis doctoral inédita, Universidad de Burgos, 2017.

${ }_{31}$ CONTCEPI: op. cit., p. 39. 
INDIGENISMO, EDUCACIÓN COLONIAL Y ETNOEDUCACIÓN MARTHA LUCÍA OROZCO GÓMEZ, M. ${ }^{a}$ DOLORES FERNÁNDEZ MALANDA Y NARDA DIOSELINA ROBAYO FIQUE

integración de los niños, niñas y jóvenes en la comunidad, así como el modo de reconocer, comprender y afrontar las dificultades buscando soluciones que permitan la pervivencia cultural, material y espiritual de los pueblos indígenas.

Otro de los elementos de la educación propia ya enunciado anteriormente es la oralidad. Dado que las comunidades primitivas no tuvieron escritura, la oralidad fue el método natural, más singular y original que utilizaron para transmitir sus conocimientos de padres a hijos, de generación en generación, y conservar de esta manera el conjunto de saberes ancestrales, como un legado para sus descendientes, sistema que ha llegado hasta nuestros días, como se manifiesta en los relatos contados por los abuelos sobre leyendas del folklore nacional o sobre situaciones anecdóticas vividas por ellos. Sapiencias comunicadas mediante relatos, leyendas, cantos y demás suponen una forma de traer el pasado al presente y proyectarlo al futuro; según el pensamiento indígena, es revivir los acontecimientos pasados por medio de la palabra.

En la transmisión oral se hace relevante el archivo de conocimientos consignados en la memoria como un receptáculo de la historia, como uno de los dispositivos más definidos para conservar la historia milenaria de los pueblos. Este archivo mental facilita el aprendizaje, puesto que asimila la vivencia y le da significado a los conocimientos.

Por otra parte, en el aprendizaje es fundamental la relación directa con el objeto facilitador de conocimiento, que permite la adquisición de experiencias propias, como se observa en el desarrollo de los trabajos en la chagra (o huerto familiar), donde se practican valores como la solidaridad y la colaboración, ya que se cuenta con la participación de familiares y amigos de las familias, y se manifiestan la reciprocidad y la generosidad al compartir en forma afectuosa algunos alimentos y bebidas. Estos valores en conjunto son algunos de los principios característicos de organización de las comunidades indígenas ${ }^{32}$.

Es importante resaltar la trascendencia que tienen las autoridades tradicionales en la educación como poseedores de los conocimientos ancestrales y de la sabiduría para dirigir el «Plan de vida», pues conservan en su memoria toda la historia de la cultura del pueblo y el conocimiento de la relación de la comunidad con la naturaleza.

Los médicos tradicionales, como personas importantes integrantes de las autoridades mayores, desempeñan un papel fundamental en la educación, por cuanto se han formado en el quehacer de la medicina tradicional que se ha constituido con base en los conocimientos aportados por la historia, la tradición y la experiencia. En su relación con la naturaleza y el mundo espiritual son poseedores de conocimientos y técnicas milenarias para tratar a los pacientes de forma integral, esto es, cuerpo, alma y espíritu. Consideran la relación que existe entre los tres elementos que constituyen el ser humano según sus creencias, son conocedores de las propiedades de las diferentes plantas y su aplicación y utilizan medicamentos naturales.

32 Robayo Fique, Narda D.: op. cit. 
Las ideas anteriores se ajustan a las concepciones de las enseñanzas certificadas por el SEIP cuando afirma que las pedagogías propias contribuyen al fortalecimiento integral de las culturas de los pueblos indígenas y se desarrollan a partir de las experiencias propias de cada comunidad, conforme a sus cosmovisiones y realidades. Su contribución especial reside en el papel determinante atribuido dentro de la comunidad a las autoridades mayores, a los médicos tradicionales o a la familia... sin olvidar la relación con una naturaleza investida de sabiduría y unos procesos, métodos y contextos de aprendizaje basados en la consulta y los acuerdos establecidos por cada pueblo ${ }^{33}$.

Los aprendizajes también se obtienen en diferentes escenarios, cada uno de los cuales contiene información valiosa que alimenta el archivo mental milenario de la comunidad y de los individuos que sin pertenecer a la misma se relacionan con sus miembros, les observan, investigan, interpretan y/o reciben sus influencias y que, por tanto, son parte de los procesos pedagógicos. Algunos de estos escenarios son los distintos espacios de socialización, los signos y elementos de la naturaleza, los fenómenos cósmicos, la chagra, los mitos y rituales, los sueños y visiones, la transmisión oral generacional de los saberes ancestrales y los conocimientos de la medicina tradicional ${ }^{34}$.

Según señala el SEIP, ha sido muy difícil asumir la sustitución violenta de la cultura milenaria por la cultura occidental, al ser impuestos modelos de desarrollo, sistemas de vida, concepciones de valores diferentes a su sociedad y a su naturaleza, elementos culturales que han sido interiorizados por los indígenas sometidos a la aculturación y negación de sus propios usos y costumbres, y que en la actualidad son una debilidad para afrontar la incierta situación en que viven.

Una forma de demostrar esta negación es analizar el impacto ocasionado por la imposición de la escuela, sistema completamente ajeno a la naturaleza indígena. Sin embargo, reconocen que se han utilizado algunos elementos proporcionados por ella para la defensa de la cultura y derechos en el proceso de la lucha indígena $^{35}$. La educación oficial les ha aportado instrumentos jurídicos para exigir sus derechos y respeto por su cultura. En el proceso de la resistencia indígena, los logros obtenidos se deben, en parte, al uso de herramientas que el Estado les ha brindado desde la educación oficial.

Sin embargo, la educación oficial vulnera la estructura de los saberes de los pueblos indígenas cuando obliga a un enfrentamiento cultural entre lo propio y lo impuesto, lo local y lo ajeno, enfrentamiento que se presenta cuando los poderes locales y los estatales, a través de sus representantes, quieren apropiarse del tiempo y los espacios educativos y las relaciones sociales y culturales de la comunidad para impartir a los escolares sus conocimientos, primando los saberes de una cultura occidental que menosprecia el saber tradicional.

Los indígenas rechazan la educación oficial porque históricamente ha denostado la tradición oral frente a la escrita. El conjunto de saberes de transmisión oral

33 CONTCEPI: op. cit.

34 Ibidem.

35 Ibidem, p. 25. 
INDIGENISMO, EDUCACIÓN COLONIAL Y ETNOEDUCACIÓN MARTHA LUCÍA OROZCO GÓMEZ, M. ${ }^{a}$ DOLORES FERNÁNDEZ MALANDA Y NARDA DIOSELINA ROBAYO FIQUE

han sido reemplazados por cartillas suministradas por las instituciones educativas que dificultan la comprensión de la lectura, debido a que los contenidos muchas veces carecen de significación para la mente indígena. Esto es contrario al método tradicional, donde el aprendizaje tiene significación debido a las evidencias percibidas en el contacto con los fenómenos naturales y en la forma de interpretar las lecturas que hacen de los diferentes elementos y mensajes aprehendidos de la naturaleza.

Es una realidad que el español es un idioma que se ha ido imponiendo progresivamente en las comunidades indígenas debido al encuentro de estas con los blancos, por diferentes motivos que ya se han tratado. A pesar de que los movimientos indígenas luchan por la defensa de sus lenguas y culturas propias, la presencia de la televisión o los servicios de internet, subsidiados por el Estado, junto con el proceso de evolución natural de las lenguas, permiten prever la extinción de las lenguas nativas de muchas comunidades, incluidas las del Trapecio amazónico.

Respecto a la religión se puede pensar en el mismo proceso de la lengua, puesto que la nueva generación está siendo sustraída de sus principios religiosos ancestrales por la doctrina católica y otros movimientos cristianos que han llegado a las diferentes regiones y han sido aceptados por algunas personas de la comunidad. Sin embargo, se consideran muy arraigados los principios de la mitología.

Igualmente, el SEIP toma como principal herramienta la investigación para la recuperación de los saberes y conocimientos tradicionales depositados en los mayores, los médicos tradicionales, los mitos, los ritos y demás prácticas culturales, en el marco de la interacción armónica entre y con la naturaleza, buscando la generación y sostenibilidad de los modelos de bienestar de la comunidad, como garantía de la soberanía alimentaria y la construcción de procesos interculturales.

Por otra parte, las organizaciones indígenas consideran que el sistema educativo oficial presenta limitaciones para los pueblos indígenas, pues no se ajusta a la visión que estos tienen de educación, la cual no se reduce a los conocimientos impartidos en un aula de clase, sino que cuenta con varios espacios reales que comunican a los alumnos con la naturaleza y la comunidad, con metodologías diferentes, mediante las cuales adquieren conocimientos, valores y pautas de convivencia, desarrollan habilidades y destrezas que les permiten desenvolverse adecuadamente en la vida y relacionarse armónicamente con su medio natural, familiar y comunitario.

Debido a la histórica relación con la cultura oficial y a los cambios ocasionados por la globalización y la modernidad, son conscientes de que no están solos y que han pasado a formar parte de este universo, por consiguiente, ven que es indispensable relacionarse con otras culturas, establecer un diálogo de saberes, compartir los conocimientos y prácticas que conduzcan a la complementación y favorecimiento de sus culturas, para un mejor desarrollo de las comunidades, y así lograr ser sujetos de consideración, respeto y entendimiento entre las respectivas sociedades. Por esta razón, las organizaciones indígenas encuentran en la educación el elemento infalible para formarse y establecer relaciones con otras culturas. 
Para los pueblos indígenas, la educación es un proceso autónomo, un espacio desde donde se construye comunidad, se perfilan los dirigentes, se construye la mentalidad crítica y se orienta a las personas para ser artífices de su proyecto de vida.

Es así como, desde el Concejo Regional Indígena del Cauca (CRIC), una de las organizaciones indígenas mejor consolidadas, se construye desde hace 30 años el Programa de Educación Bilingüe Intercultural (PEBI), «una propuesta de educación pertinente a los contextos culturales de las comunidades indígenas, a sus problemáticas y a sus necesidades y a sus proyecciones y esperanzas» ${ }^{36}$. El PEBI se convierte en una clara estrategia de reapropiación y modernización de las comunidades indígenas, favoreciendo su acercamiento y participación en contextos nacionales e internacionales en igualdad de condiciones, por cuanto ha fortalecido sus conocimientos, sabiduría y autonomía, con capacidad de gestión que los faculta para cumplir con la responsabilidad que les ha asignado la sociedad mayoritaria.

\section{Conclusiones}

Los pueblos indígenas originarios han pervivido a lo largo del tiempo, entre otras cosas, por el convencimiento en sus creencias y el amor que tienen por todo cuanto les rodea y les permite vivir. La lucha por mantener y rescatar su cosmovisión, que entraña una relación consciente con la Pachamama y todo ser viviente, la lucha por su territorio y por conservar su identidad a través de la educación nos merece un profundo respeto y admiración.

Investigar, vivir, cohabitar con otras culturas, otras formas de ver y entender la vida tiene la enorme virtud de suscitar esperanzas en lograr un mundo donde todos tengamos cabida, y, más allá de esto, donde la raza humana pueda sobrevivir a su propia autodestrucción, por agotamiento de los recursos no renovables. En este sentido, nos referimos al Sumak Karesay como una concepción del desarrollo que surge de la cosmovisión indígena y se contrapone al modelo de desarrollo hegemónico occidental, la crematística. Esta concepción filosófica, anclada en fuertes saberes ancestrales, nos enseña que hay otras alternativas a la relación existente entre los seres humanos y la naturaleza y entre ellos mismos. Esto se ha convertido, gracias a sus luchas, en un baluarte que les ha permitido permanecer hasta hoy. Es interesante descubrir, a medida que se profundiza en este modo de ver la vida, que las luchas por mantener sus raíces no significa una idealización del pasado ni un estancamiento en este, sino que se busca una complementariedad entre las propias raíces y otras culturas que les rodean.

En la unión entre su cosmovisión y la lucha por una educación propia se ha encontrado una herramienta fundamental para adaptarse a nuevas situaciones manteniendo su identidad, debido a que «dentro del universo indígena la educación no es una función social autonomizada en una instancia escolar, es un asunto continuo cuya responsabilidad recae en todos los miembros de la comunidad.

36 Bolaños, Graciela: op. cit., p. I62. 
INDIGENISMO, EDUCACIÓN COLONIAL Y ETNOEDUCACIÓN MARTHA LUCÍA OROZCO GÓMEZ, M. ${ }^{a}$ DOLORES FERNÁNDEZ MALANDA Y NARDA DIOSELINA ROBAYO FIQUE

Igualmente, el principio fundamental del proceso de enseñanza-aprendizaje es el aprender haciendo, el entorno es el libro básico, la sabiduría ancestral el contenido, y el niño co-partícipe del proceso» ${ }^{37}$.

Es por ello que las comunidades indígenas, representadas por diferentes organizaciones, vienen reclamando autonomía para poder practicar su propia educación, pues esta representa el rescate y mantenimiento de su cultura. El lograr en algunos países (Colombia o Bolivia, por ejemplo) un modelo etnoeducativo, es un avance importante, pero finalmente no satisface las demandas de las comunidades, ya que no se logra una verdadera educación diferenciada y autónoma.

Un gran salto se produce en 2007 con la declaración de las Naciones Unidas sobre los Derechos de los pueblos indígenas, que fortalece y apoya la creación del Sistema Educativo Indígena Propio (SEIP) introducido en los centros a partir del diseño del Proyecto Educativo Comunitario (PEC); en estos proyectos se plasma la política educativa de los pueblos indígenas, siendo una herramienta base para los «Planes de vida», y evidenciando los avances que se han ido consiguiendo.

Se tiene claro que estas propuestas educativas no solo deben ayudar a la conservación de la propia cultura, sino también preparar para afrontar el mundo actual. Recogemos, como aspectos que demuestran la integralidad de sus concepciones, los componentes que consideran importantes dentro su sistema educativo y que son considerados como partes de una formación integral: la familia, la naturaleza y los saberes ancestrales. En este proceso la oralidad, elemento clave dentro de sus tradiciones, juega un papel importante. De la misma manera, no se puede olvidar la relación con los espíritus y los fenómenos astronómicos, todo ello integrado en los quehaceres cotidianos. En otras palabras, el indigenismo en general no entiende la educación sin una relación directa con el objeto que se desea conocer, y este debe abarcar desde el conocimiento propiamente dicho hasta los valores. Esto es, cuerpo, alma y espíritu.

Las pedagogías propuestas están imbricadas en sus cosmovisiones y realidades, y cuentan con la contribución directa de los mayores y la familia en general. Es importante comprender que las comunidades indígenas no están cerradas a aprender de otras culturas, a entenderlas y a complementarse, y que entienden su educación como un elemento de gran importancia para mantenerse y establecer otras relaciones.

La escuela oficial sustituye sus valores, lengua, religión, y luchan contra ello, pero a la vez utilizan estos propios aprendizajes para incorporarlos a su defensa, por ejemplo, el aprendizaje de temas relacionados con el derecho les ha sido de gran utilidad.

Varios Estados americanos han dado autonomía para que las culturas indígenas organicen sus sistemas, sin embargo, muchos de los acuerdos no están siendo cumplidos, sobre todo en materia educativa. No se está permitiendo la educación bilingüe, debido a que muchos de los directivos de los centros, especialmente aquellos que están en manos de congregaciones religiosas, obligan al estudio del

37 Garzón Pérez, Luis Alfonso: op. cit., p. 588. 
español como lengua principal y el inglés como la segunda, y a que los contenidos trabajados desconocen, en muchos casos, las directrices planteadas en materia de inclusión de sus saberes.

Para continuar avanzando, es de suma importancia pensar en el liderazgo que han de retomar los educadores como conductores de la sociedad: investigando, presentando propuestas y proyectos para lograr que los modelos y paradigmas pedagógicos respondan a las necesidades de la población actual, que hoy enfrenta los retos de los cambios de la nueva civilización, contribuyendo a la construcción de un nuevo modelo de sociedad.

Resta camino por recorrer, no hay soluciones definitivas, pero sí nos podemos acercar poco a poco al reconocimiento real y genuino de esas otras formas maravillosas de vivir. Un nuevo y gran impulso puede surgir en 2019, declarado por la ONU como Año Internacional de las Lenguas Indígenas. Estaremos atentos a estos acontecimientos y a las nuevas oportunidades que pueda traer para seguir en el buen camino... 